\title{
Comments On Dr. J. Slawinski's Paper
}

\author{
Rupert Sheldrake, Ph.D. \\ London, England
}

I agree with Janusz Slawinski in thinking that concepts of the soul may eventually be formulated in terms of scientific hypotheses. But I think it unlikely that the current concepts of science, including electromagnetism, are adequate for this purpose. These are the main obstacles I find in the way of following his approach:

1. Not only does "all living matter from cell organelles to man" create electromagnetic (EM) fields, but so does all matter, living and nonliving. Not only does "some part of the field extend beyond the material boundaries of the organism, creating the so-ealled electromagnetic aura," but part of the field extends around everything, living and nonliving. Not only do living organisms give off EM radiation, but so do inanimate objects such as light bulbs and even rocks. For example, the latter can be photographed at night on the basis of their infrared photon emission. So the presence of EM fields in and around organisms and the emission of photons do not necessarily reveal anything special about life, nor imply that EM fields are associated with consciousness. EM fields are associated with all matter; all material systems can emit photons; and all material changes are associated with changes in the EM field. That is what the standard theories of physics tell us, and so it is not surprising or especially significant that changes in organisms and brains are associated with EM effects, including photon emission.

2. Patterns of change in any material system, be it a DNA molecule or a motor car, are associated with patterns of EM change that could be considered to contain spatiotemporal information. The patterns of EM radiation from the bonnet of a car depend on the activities of the

Dr. Sheldrake was formerly Director of Studies in Biochemistry and Cell Biology at Clare College, Cambridge, and Consultant Plant Physiologist at the International Research Institute in India. Requests for reprints should be sent to Dr. Sheldrake at 20 Willow Road, Hampstead, London, NW3 ITJ, England. 
engine, including its electrical system, spark plugs, etc., but the EM fields around the engine do not in themselves organize or control the car. No more do data showing EM changes associated with living organisms or brains "reveal that electromagnetic force-fields mold and control the internal biological milieu within and outside of which life manifests itself." No amount of measurement or observation of the EM changes around a car engine could prove that the EM field is a "supreme factor" in organizing the car or in "forming its essence." Of course, electromagnetism is essential for cars in a general sense, and also in the particular sense that they contain electrical mechanisms, but the EM field is not supreme in controlling where the car goes, or how it is driven, or how it was designed and made in the first place.

3. Many chemical reactions occur in damaged and dying cells, as enzymes and their substrates are released from intracellular compartments, and as hydrolytic and oxidative processes occur. A familiar example is the browning of apples after being cut. Many of those changes release heat and involve excited molecules that may emit photons. As Slawinski indicates, a death flash as a result of such degradative processes could be thought of as an epiphenomenon of no particular significance. His reasons for thinking otherwise do not seem to me persuasive.

4. If a car ends up in a crash, it may emit a very spectacular "death flash" as it burst into flames. The radiation will carry information, depending on which parts burn first, what temperature they burn at, where they are in relation to each other, and so on. Like all radiation, this would move at the speed of light; the photons would be potentially immortal; they would just be. But that would not prove that the car was conscious or that its consciousness became separated from it when it burst into flames, entering another dimension.

5. Even if we assume that a coherent pattern of information is encoded in the death flash emitted by a brain, and that the pattern expands at the speed of light and just is, I find it impossible to imagine how such a pattern could form the basis for the continuation of life beyond the death of the physical body. In any case, the pattern would be disrupted as soon as anything got in the way and absorbed some of the photons, just as the pattern of light going out of the window of my room at present is rapidly degraded.

6. I cannot imagine how Slawinski's hypothesis can be tested, and I do not think that research on low-level luminescence and other radiation of living systems could possibly provide "a realistic basis for dealing with the problems of an afterlife." 